\title{
GENERATION AND COMPARISON OF TLS AND SFM BASED 3D MODELS OF SOLID SHAPES IN HYDROMECHANIC RESEARCH
}

\author{
R. Zhang a,b, D. Schneider ${ }^{\mathrm{a}, *}$, B. Strauß ${ }^{\mathrm{c}}$ \\ a Institute of Photogrammetry and Remote Sensing, Technische Universität Dresden, Germany - \\ rongchun.zhang@mailbox.tu-dresden.de, danilo.schneider@tu-dresden.de \\ b School of Earth Sciences and Engineering, Hohai University, Nanjing, China \\ ${ }^{c}$ Institute of Hydraulic Engineering and Technical Hydromechanics, Technische Universität Dresden, Germany - \\ Benjamin.strauss@mailbox.tu-dresden.de
}

\author{
Commission V, WG V/5
}

KEY WORDS: Terrestrial Laser Scanning, Structure-from-Motion, 3D Model, Volume Calculation, Hydromechanics

\begin{abstract}
:
The aim of a current study at the Institute of Hydraulic Engineering and Technical Hydromechanics at TU Dresden is to develop a new injection method for quick and economic sealing of dikes or dike bodies, based on a new synthetic material. To validate the technique, an artificial part of a sand dike was built in an experimental hall. The synthetic material was injected, which afterwards spreads in the inside of the dike. After the material was fully solidified, the surrounding sand was removed with an excavator. In this paper, two methods, which applied terrestrial laser scanning (TLS) and structure from motion (SfM) respectively, for the acquisition of a 3D point cloud of the remaining shapes are described and compared. Combining with advanced software packages, a triangulated 3D model was generated and subsequently the volume of vertical sections of the shape were calculated. As the calculation of the volume revealed differences between the TLS and the SfM 3D model, a thorough qualitative comparison of the two models will be presented as well as a detailed accuracy assessment. The main influence of the accuracy is caused by generalisation in case of gaps due to occlusions in the 3D point cloud. Therefore, improvements for the data acquisition with TLS and SfM for such kind of objects are suggested in the paper.
\end{abstract}

\section{INTRODUCTION}

TLS, emerged in the mid-1990s, allows capturing accurate 3D models of an object in short time, to be used for several purposes. TLS is an active and non-contact measurement technique which can obtain the spatial coordinates of an object with high speed and accuracy by measuring the time-of-flight of laser signals. High temporal resolution, high spatial resolution and uniform accuracy make the TLS data rather appropriate for many applications, such as topography (Buckley, 2008, Eltner \& Baumgart, 2015), monitoring (Rosser, 2005), archaeology (Lerma, 2010), city modeling and civil engineering ( $\mathrm{Pu}$, et al., 2009). SfM, as a primary technique of computer vision to reconstruct 3D scene geometry and camera motion from a set of images of a static scene, has been applied in more and more areas, including geomorphology (Eltner \& Schneider, 2015, Stöcker et al., 2015), medical science (Reiter, et al., 2016), archaeology (Opitz, et al., 2016) and cultural heritage (Mu, 2015). SfM has the potential to provide both a low cost and time efficient method for collecting data on object surface (Armistead, 2013). It's different from traditional photogrammetry, SfM technique neither needs any prior knowledge about camera positions and orientation, nor targets with known 3D coordinates, all of which can be solved simultaneously using a highly redundant, iterative bundle adjustment procedure, based on a database of features automatically extracted from a set of multiple overlapping images (Snavely, 2008; Westoby, et al., 2012)

The aim of a current study at the Institute of Hydraulic Engineering and Technical Hydromechanics at TU Dresden is to develop a new injection method for quick and economic sealing of dikes or dike bodies, based on a new synthetic material. In particular, a solution has to be found which enables a subsequent and permanent internal sealing of dikes. Thereby, weakness in the dam as well as in the connection area between soil and the solid construction should be fixed by prompt injections of this synthetic material into the dike. The advantage of this method is the rapid applicability in places, which are hard to reach for machines.

The remaining solidified shapes after removing the surrounding sand have to be analysed and interpreted in order to assess the success of the new injection method. For this purpose, the shapes have to be virtually preserved in terms of a 3D model which enables subsequent quantitative and qualitative analysis.

For the 3D recording of these shapes two methods are described and compared in this paper. The first method is terrestrial laser scanning applying a RIEGL LMS-Z420i and the second method is structure-from-motion applying a Nikon DSLR and processing with AgiSoft PhotoScan. Then a triangulated 3D model was generated and subsequently the volume of vertical sections of the dike were calculated by using Geomagic Studio. Thereby it should be investigated which amount of the injected synthetic material is present in each height section after solidification. As the calculation of the volume revealed significant differences between the two 3D models built from TLS and from SfM respectively, a thorough qualitative comparison between the two models as well as a detailed accuracy assessment are presented. The main influence of the accuracy is caused by generalisation in case of gaps due to occlusions in the 3D point cloud. Therefore, improvements for the data acquisition with TLS and SfM for such kind of objects are suggested in the paper. 
In recent years, similar comparisons between TLS and SfM in different fields were proposed. In the area of cultural heritage, a range of comparisons in accuracy, density, time consuming about the two techniques were carried (Smith, et al., 2015; Fonstad, et al., 2013). In geomorphology research, Anette made a quantitative measurement and comparison of soil erosion with DTM respectively generated from TLS and SfM (Eltner et al., 2015). Skarlatos \& Kiparissi (2012) compared TLS and SfM with three tests to evaluate the process as well as data density, quality, registration and methodology.

\section{DATA ACQUISITION}

\subsection{The design of the project}

To validate the new technique presented by the Institute of Hydraulic Engineering and Technical Hydromechanics at TU Dresden, an artificial part of a sand dike was built in an experimental hall. The synthetic material was injected, which afterwards spreads in the inside of the dike. After the material was fully solidified, the surrounding sand was removed with an excavator. Only the solid shapes were remained, which look like a wall with a lot of curvatures or like connected columns (Fig. 1). The size of the remaining shapes was approximately $1.4 \mathrm{~m} \times 0.6 \mathrm{~m}$ $\times 1.6 \mathrm{~m}$.

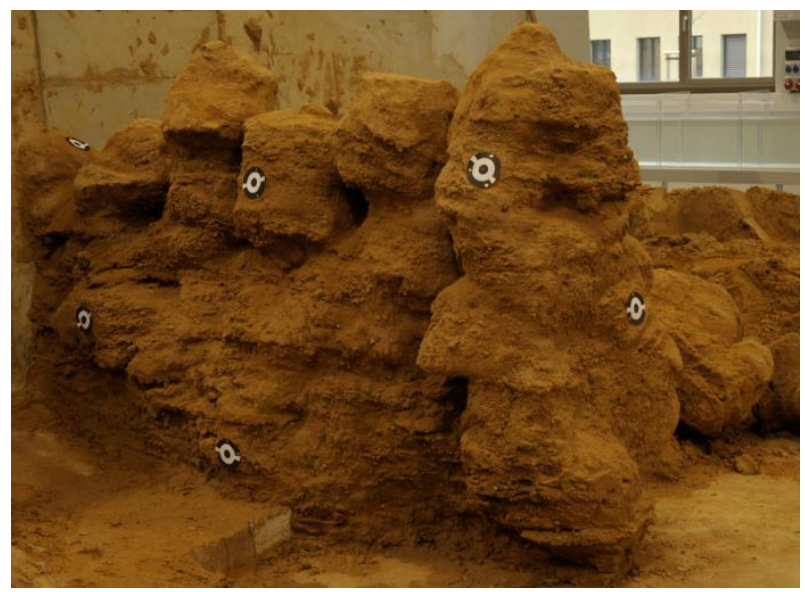

Figure 1. Picture of the remaining shapes

\subsection{TLS Data Acquisition}

In this paper, the terrestrial laser scanner system, RIEGL LMSZ420i (Fig. 2), was selected for acquiring TLS data of the solid shapes. The system is a rugged and fully portable sensor specially designed for the rapid acquisition of high-quality $3 \mathrm{D}$ data in the field, the measurement rate of which is up to 11000 points per second, the minimum range is 2 meters, and the field of view is up to $80^{\circ} \times 360^{\circ}$. The accompanying operating and processing software, RiSCAN PRO, provides various functions of registration, post processing and export.

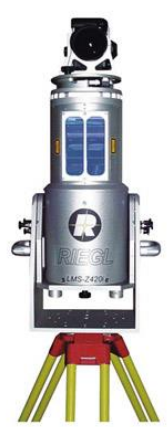

Figure 2. RIEGL LMS-Z420i

A set of cylinder targets were deployed to register the TLS data as tie points. Scans of the remaining shapes were acquired from five positions (Fig. 3). The distance between the object and different positions was in the range of 4-6 meters. The resolution (point spacing on the object surface) was set to $0.0024 \mathrm{~mm}$ (@4m) resp.0.0036mm (@6m).

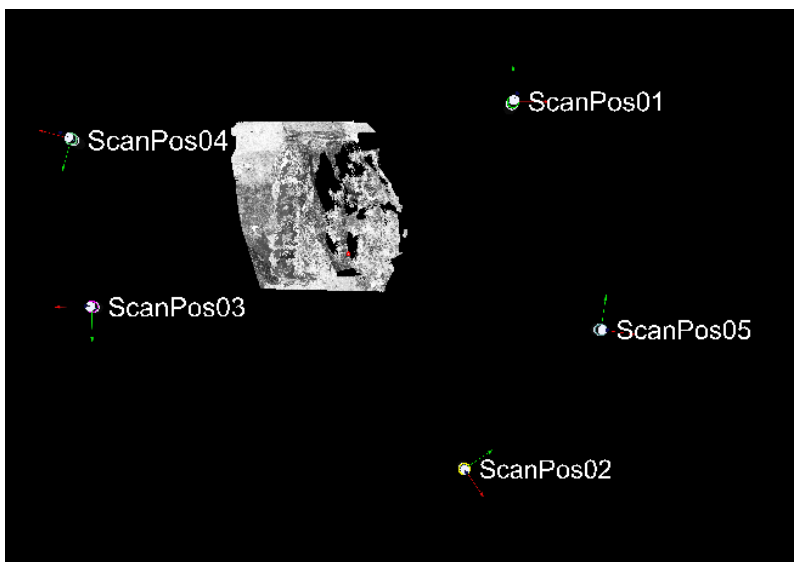

Figure 3. The positions of the scanner

\subsection{SfM Data Acquisition}

SfM is a process of estimating the structure of spatial configurations from the sequence of $2 \mathrm{D}$ projections. The advantage of SfM technique is its simplicity, i.e., a 3D model can be constructed with digital images without knowledge of camera locations and optics information. SfM model can be generated with any set of photographs which contain sufficient image overlap and adequate quality.

Moreover, the quality of the generated dense point cloud data largely depends on both the quality of all images, including resolution, and the clarity of the shape textures.

In this paper, a camera Nikon D700 combined with a $50 \mathrm{~mm}$ lens, was used for image acquisition. Because the dark lightning conditions were challenging and the use of the flash light might be disadvantageous for the SfM processing it was necessary to find a compromise between ISO, exposure time and aperture settings. In this measurement, the ISO was set to 800 , the shutter was set to $1 / 50 \mathrm{~s}$ and an aperture of $\mathrm{f} / 9$ was choosen. Considering the special shape of the object and to avoid occlusion, more than two hundred photos were taken sequentially around the shape at equal small angles interval and similar distance to the object. And three groups of images were divided from top to bottom. The overlap of both, vertical and horizontal direction have reached more than $60 \%$. After quality estimation, 152 images were used for dense point cloud generation. 


\section{DATA PROCESSING}

\subsection{TLS Data Processing}

At first, each scan position has to be registered in a common project coordinate system using registration targets. The number of used targets was 27, and the standard deviation of the registration error was ca. $2 \mathrm{~mm}$ for all scan positions. The combined TLS point cloud after registration is shown in Fig. 4.

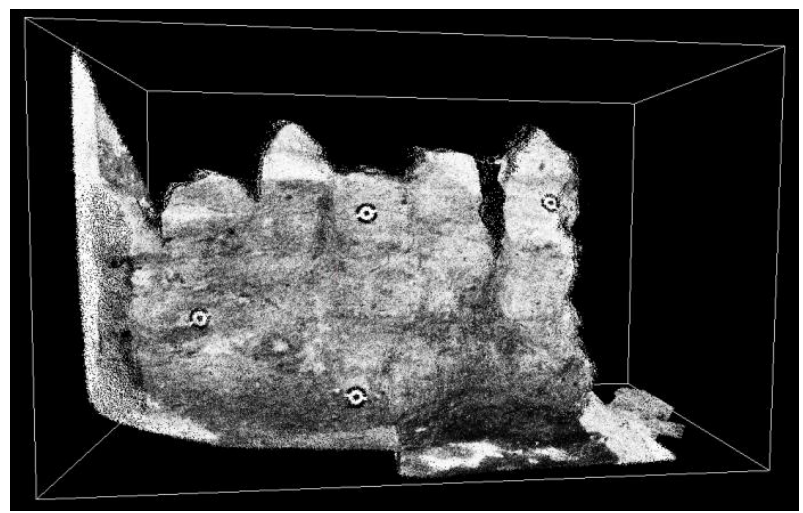

Figure 4. TLS point cloud after registration of 5 positions

\subsection{SfM Data Processing}

For the SfM processing the software Agisoft PhotoScan was used Here, identifying features in individual images is an initial processing step for image alignment. By extracting features in each image that are invariant to the image scaling and rotation, the key points can be obtained automatically. The number of key points in an image mainly depends on image texture and resolution (Westoby, et al., 2012). In this paper, 18,574 tie points were extracted and a sparse point cloud model was built. Furthermore, the camera position and orientation for each image were determined (Fig. 5).

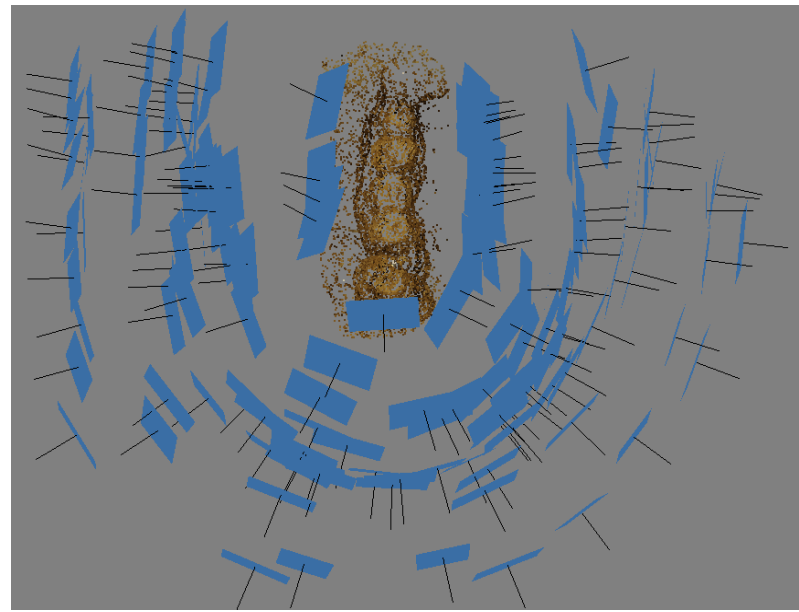

Figure 5. Sparse point cloud and imaging configuration (top view, Screenshot Agisoft PhotoScan)
The dense point cloud consists of 7,397,846 points. The high density point cloud as well as the corresponding colour information are visualized in Fig. 6.

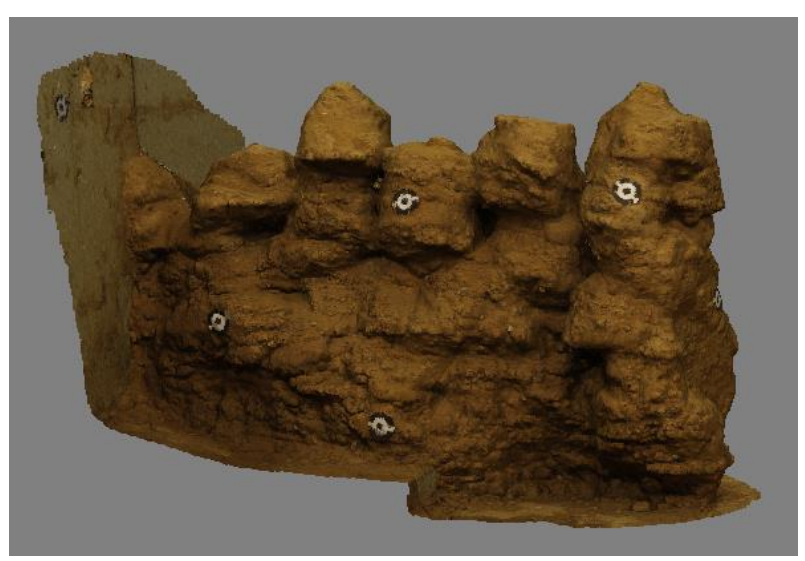

Figure 6. Dense point cloud model

\subsection{Data registration}

The dense point cloud model is still defined in an arbitrary coordinate system with an arbitrary scale. In order to provide a correct scaling of the model for further volume measurements and comparison with the TLS 3D model the model has to be transformed into the same coordinate system as the TLS 3D model. Afterwards the two point clouds can be imported in Geomagic Studio for further processing.

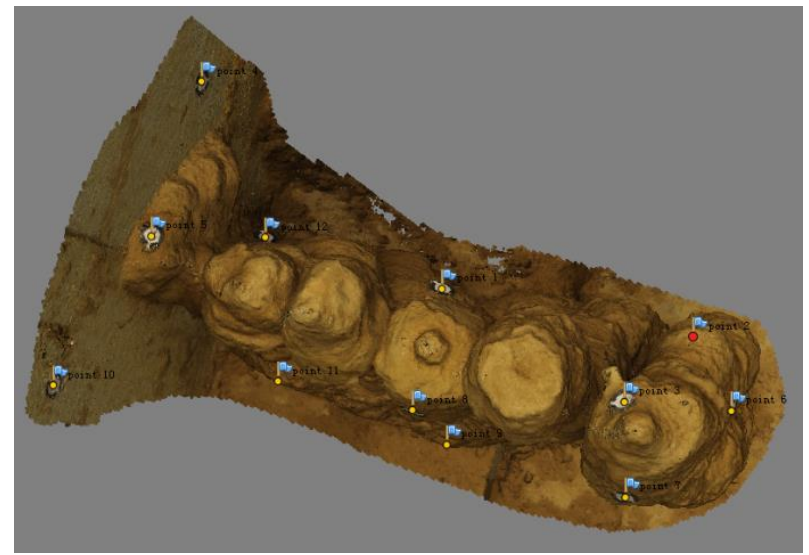

Figure 7. Distribution of markers and numbering (top view)

For this purpose, twelve pairs of corresponding markers were selected interactively in order to register the SfM point cloud in the same coordinate system as the TLS point cloud and to provide the correct scaling (Fig. 7). In this paper, the standard deviation of all marker points was less than $2 \mathrm{~mm}$.

After finishing all the above processing steps, both the TLS 3D model and the SfM 3D model are given in the same reference system (Fig. 8). 


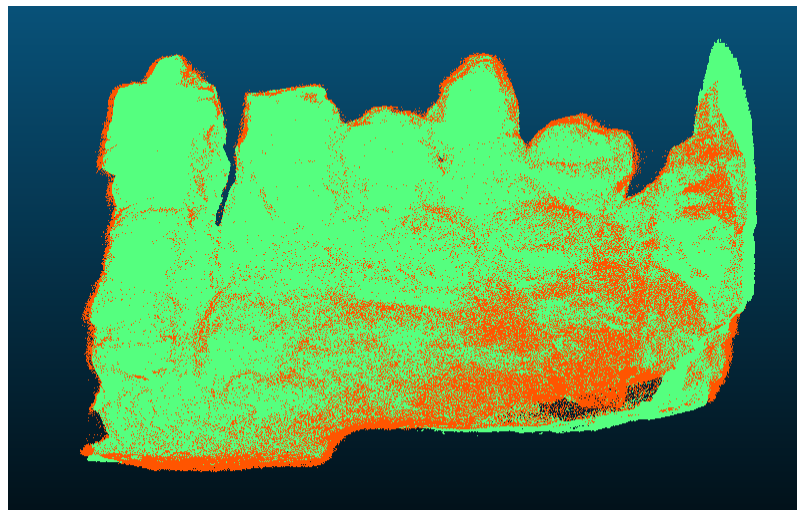

Figure 8 TLS data (green) and SfM data (red)

\section{RESULTS AND DISCUSSION}

Two 3D point cloud data of the remaining shape obtained by TLS and SfM respectively could be imported into Geomagic Studio software for further reconstruction and volume calculation.

\subsection{Triangulation and further processing}

Since much noise existed in the point cloud data from TLS, an interactive editing was necessary. And the number of points obtained with TLS was 2,773,039. For a better view as well as easier manual editing to the point cloud, point normals were calculated. Subsequently, a series of processing steps, including the elimination of disconnected components as well as outliers, the reduction of noise, and uniform sample, were finished. Afterwards, a triangulated 3D model from TLS point cloud data was built (Fig. 9, left).
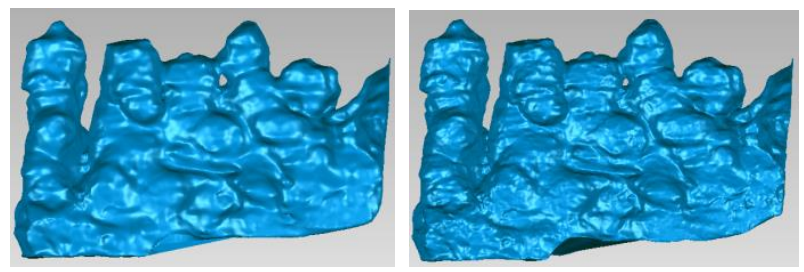

Figure 9. Point cloud from TLS (left) and SfM (right)

On the other hand, for the point cloud data of the remaining shapes from SfM, the number of points was about $4,150,118$, which means more than double number of points in comparison to the TLS data $(1,604,448$ points). Finally, a triangulated 3D model from SfM point cloud data was generated, too (Fig. 9, right). From Figure 9, it seems that the 3D model from SfM has a more detailed structure, comparing with the one from TLS. This is mainly because the TLS data contained more noise which have to be deleted by combination of automatic smoothing and some interactive work, which would have different visual effects on both the details and texture of the model.

\subsection{Volume calculation}

The shape was separated into 8 sections to compare volumetric calculations, and the extent of the vertical sections was defined with $0.2 \mathrm{~m}$ (Fig. 10). As it was difficult to determine the exact bottom of this object for both TLS and SfM 3D model, this paper only made a comparison to the first 7 sections from top to down.

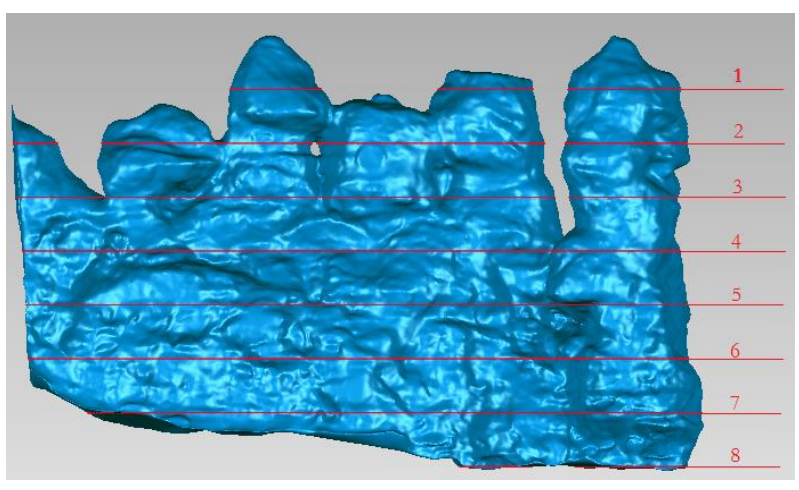

Figure 10. 3D model of the shapes and $0.2 \mathrm{~m}$-sections

The volume for each section as well as the total volume of TLS 3D model and SfM 3D model are shown in Table 2.

\begin{tabular}{|c|c|c|c|c|}
\hline Section $\mathrm{i}$ & $\Delta \mathrm{H}(\mathrm{m})$ & $\mathrm{V}_{\mathrm{TLS}-(\mathrm{i})}\left(\mathrm{m}^{3}\right)$ & $\mathrm{V}_{\mathrm{SfM}-(\mathrm{i})}\left(\mathrm{m}^{3}\right)$ & $\Delta \mathrm{V}_{\mathrm{i}}\left(\mathrm{m}^{3}\right)$ \\
\hline 1 & 0.020 & 0.015 & 0.025 & -0.010 \\
\hline 2 & 0.020 & 0.101 & 0.103 & -0.002 \\
\hline 3 & 0.020 & 0.162 & 0.158 & 0.004 \\
\hline 4 & 0.020 & 0.191 & 0.195 & -0.004 \\
\hline 5 & 0.020 & 0.236 & 0.237 & -0.001 \\
\hline 6 & 0.020 & 0.262 & 0.269 & -0.007 \\
\hline 7 & 0.020 & 0.266 & 0.265 & 0.001 \\
\hline Total & & 1.233 & 1.252 & -0.019 \\
\hline
\end{tabular}

Table 2. Calculated volume for each section and total volume

\subsection{Qualitative comparison of SfM and TLS results}

Comparing the volume results of the two models, SfM is superior in terms of data acquisition time, texture clarity of objects and modelling quality.

The two point clouds have different point densities. Therefore, besides further reasons mentioned in the following section, there are differences between the volume results. In Table 2 it is shown that the volume difference between the two models is in a range between 0.001 and $0.010 \mathrm{~m}^{3}$. The total $\mathrm{SfM}$ volume is larger than the volume calculated based on TLS data. This might be caused by errors in remaining scaling errors as well as by the smoothing of the TLS data which might also remove fine details of the surface.

The comparison of 3D model generation and the volume calculation with TLS and SfM shows that errors occur within the procedure of data acquisition as well as the data processing, which largely affects the accuracy of the generated models.

For the research objects in this paper, the extremely irregular shape made the TLS data acquisition more difficult: occlusion would be more with less scan positions. However, it would cost more time if more scan positions were set.

On the other hand, both the low texture (homogenous texture) of the objects and the constraint condition of low light inside the experimental hall made a bad effect on the quality of images used for SfM. Beyond that, however, the accuracy of dense point cloud generation also depended on the image configuration and the horizontal as well as vertical overlap between images. 


\section{CONCLUSIONS}

In this paper, a comparison between TLS and SfM was presented by volume calculation. And it can be concluded that in order to avoid occlusion as much as possible and get more accurate results, more attention should be paid to the deployment of scan positions, including the number as well as the location.

For SfM, although images can be acquired at a relative random way, enhancing light condition, taking an appropriate imaging configuration, and keeping enough overlap between images are all helpful to guarantee complete cover of research area, which would improve the accuracy dense point cloud generation.

Besides, as the remaining shapes were partly covered with sand which provides only low texture, it was very difficult to extract some evident feature points. Setting a series of markers on the body of the objects was a feasible way to correspond TLS 3D model to SfM 3D model.

\section{ACKNOWLEDGEMENTS}

The authors would like to thank the Institute of Photogrammetry and Remote Sensing as well as the Institute of Hydraulic Engineering and Technical Hydromechanics, Technische Universität Dresden, Germany for their support of this research. The authors also would like to thank the China Scholarship Council and Hohai University.

\section{REFERENCES}

Armistead C C, 2013. Applications of 'Structure from Motion' Photogrammetry to River Channel Change Studies [D]. Boston College. College of Arts and Sciences.

Buckley S J, Howell J A, Enge H D, et al, 2008. Terrestrial laser scanning in geology: data acquisition, processing and accuracy considerations [J]. Journal of the Geological Society, 165(3): 625-638.

Eltner A, Baumgart P, 2015. Accuracy constraints of terrestrial lidar data for soil erosion measurement: application to a Mediterranean field plot [J]. Geomorphology, 245: 243-254.

Eltner A, Baumgart P, Maas H G, et al, 2015. Multi - temporal UAV data for automatic measurement of rill and interrill erosion on loess soil[J]. Earth Surface Processes and Landforms, 40(6): 741-755.

Eltner A, Mulsow C, Maas H G, 2013. Quantitative measurement of soil erosion from TLS and UAV data [J]. ISPRS-International Archives of the Photogrammetry, Remote Sensing and Spatial Information Sciences, 1(2): 119-124.

Eltner A, Schneider D, 2015. Analysis of Different Methods for 3D Reconstruction of Natural Surfaces from Parallel - Axes UAV Images[J]. The Photogrammetric Record, 30(151): 279299.

Fonstad M A, Dietrich J T, Courville B C, et al, 2013. Topographic structure from motion: a new development in photogrammetric measurement[J]. Earth Surface Processes and Landforms, 38(4): 421-430.

Grussenmeyer P, Landes T, Voegtle T, et al, 2008. Comparison methods of terrestrial laser scanning, photogrammetry and tacheometry data for recording of cultural heritage buildings [J]. ISPRS Arch. Photogramm. Remote Sens, 37: W5.
Lerma J L, Navarro S, Cabrelles M, et al, 2010. Terrestrial laser scanning and close range photogrammetry for 3D archaeological documentation: the Upper Palaeolithic Cave of Parpalló as a case study [J]. Journal of Archaeological Science, 37(3): 499-507.

Moons, T., 1997. Report on the Joint ISPRS Commission III/IV Workshop "3D Reconstruction and Modelling of Topographic Objects", Stuttgart, Germany http://www.radig.informatik.tumuenchen.de/ISPRS/WG-III4-IV2-Report.html (28 Sep. 1999).

Mu E, 2015. Fusion of 3D data from different image-based and range-based sources for efficient heritage recording [C]//2015 Digital Heritage. IEEE, 1: 83-86.

Opitz R S, Johnson T D, 2016. Interpretation at the Controller's Edge: Designing Graphical User Interfaces for the Digital Publication of the Excavations at Gabii (Italy) [J]. Open Archaeology, 1(1).

$\mathrm{Pu}$ S, Vosselman G, 2009. Knowledge based reconstruction of building models from terrestrial laser scanning data [J]. ISPRS Journal of Photogrammetry and Remote Sensing, 64(6): 575-584.

Reiter A, Leonard S, Sinha A, et al, 2016. Endoscopic-CT: learning-based photometric reconstruction for endoscopic sinus surgery[C]//SPIE Medical Imaging. International Society for Optics and Photonics: 978418-978418-6.

Rosser N J, Petley D N, Lim M, et al, 2005. Terrestrial laser scanning for monitoring the process of hard rock coastal cliff erosion [J]. Quarterly Journal of Engineering Geology and Hydrogeology, 38(4): 363-375.

Skarlatos D, Kiparissi S, 2012. Comparison of laser scanning, photogrammetry and SFM-MVS pipeline applied in structures and artificial surfaces [J]. ISPRS Annals of the Photogrammetry, Remote Sensing and Spatial Information Sciences, 3: 299-304.

Smith M W, Vericat D, 2015. From experimental plots to experimental landscapes: topography, erosion and deposition in sub - humid badlands from Structure - from - Motion photogrammetry $[\mathrm{J}]$. Earth Surface Processes and Landforms, 40(12): 1656-1671.

Snavely N, Seitz S M, Szeliski R, 2008. Modeling the world from internet photo collections [J]. International Journal of Computer Vision, 80(2): 189-210.

Stöcker, C., Eltner, A. \& Karrasch, P., 2015. Measuring gullies by synergetic application of UAV and close range photogrammetry - A case study from Andalusia, Spain. Catena, 132, pp. 1-11.

Westoby M J, Brasington J, Glasser N F, et al, 2012. 'Structurefrom-Motion'photogrammetry: A low-cost, effective tool for geoscience applications [J]. Geomorphology, 179: 300-314. 